\title{
Staining clinical specimens for acid-fast bacilli by means of a mechanical conveyor system
}

\author{
G. V. HEIMER, NATASHA JOSEPH, AND C. E. D. TAYLOR ${ }^{1}$ \\ From the Public Health Laboratory and Department of Microbiology, Central Middlesex Hospital, Park \\ Royal, London NW10 7NS, UK
}

SUMmARY A Cyto-Tek staining machine designed for Papanicolau staining of cervical smears has been adapted for auramine staining of smears of sputum and other clinical specimens for acid-fast bacilli. The results compared favourably with those obtained by a manual method of staining. Contamination of negative smears by acid-fast bacilli from positive smears was not found. Since this study the machine has continued to be in routine use with a considerable saving in labour.

Compared with other specialties in clinical pathology there is in microbiology, as previously indicated (Taylor, 1969), a singular lack of suitable machines for performing the more tedious and repetitive tasks although machines that assist with serological tests are now becoming more generally accepted. The staining of smears of sputum and other clinical specimens for acid-fast bacilli appears to be a procedure that lends itself particularly well to mechanisation. Not only is it tedious and timeconsuming when large numbers of slides are involved, but with a machine it should be easier to ensure that the staining is done consistently in a standardized manner.

In less developed countries pulmonary tuberculosis is a major cause of illness and death. Efforts to combat this disease require simple means for identifying infectious persons, and reliance is now being placed more on the examination of sputum for acid-fast bacilli than on such methods as mass miniature radiography and tuberculin skin testing. In such circumstances, as well as in the busier laboratories of more developed countries where tuberculosis is still prevalent, a machine that can be relied upon to stain smears of sputum and other clinical specimens for acid-fast bacilli would be a considerable asset.

Mechanical procedures for staining smears of clinical specimens on microscope slides have been

'Present address: Regional Public Health Laboratory and Department of Microbiology, Level 6, Addenbrooke's Hospital, Hills Road, Cambridge, CB2 2QW, UK

Received for publication 18 July 1977 evaluated by Cremer (1968), Burdash et al. (1975), and Clancey et al. (1976). The particular systems described by these authors require batches of slides to be immersed in reagents that are used repeatedly.

The potential advantages of using a mechanical means of staining smears of clinical specimens for acid-fast bacilli when large numbers of specimens have to be examined prompted us to investigate the Cyto-Tek staining machine ${ }^{1}$ (Figure). This machine was designed originally for continuous linear, automatic processing of smears of exfoliated cells according to Papanicolaou's staining sequence. Slides are treated individually, and each reagent, having once been used on a smear, is discarded.

\section{Material and methods}

\section{THE MACHINE}

Slides, $7.5 \times 2.5 \mathrm{~cm}$, are loaded smear-side facing downwards into a magazine whence they are transported by means of a chain conveyor in such a way that each slide is supported above and moves over a platen on to which measured amounts of each reagent are pumped sequentially to each slide in turn. The platen is divided into 14 sections of varying width, any one of which may be connected by a tube to a reservoir of reagent. Each section is separated from adjacent sections by a channel into which the reagent is discarded after use on one smear only. At the end of the staining process slides are dried by a stream of warm air and are then deposited

${ }^{1}$ Ames Company, Division of Miles Laboratories Ltd, Slough SL2 4LY, UK 


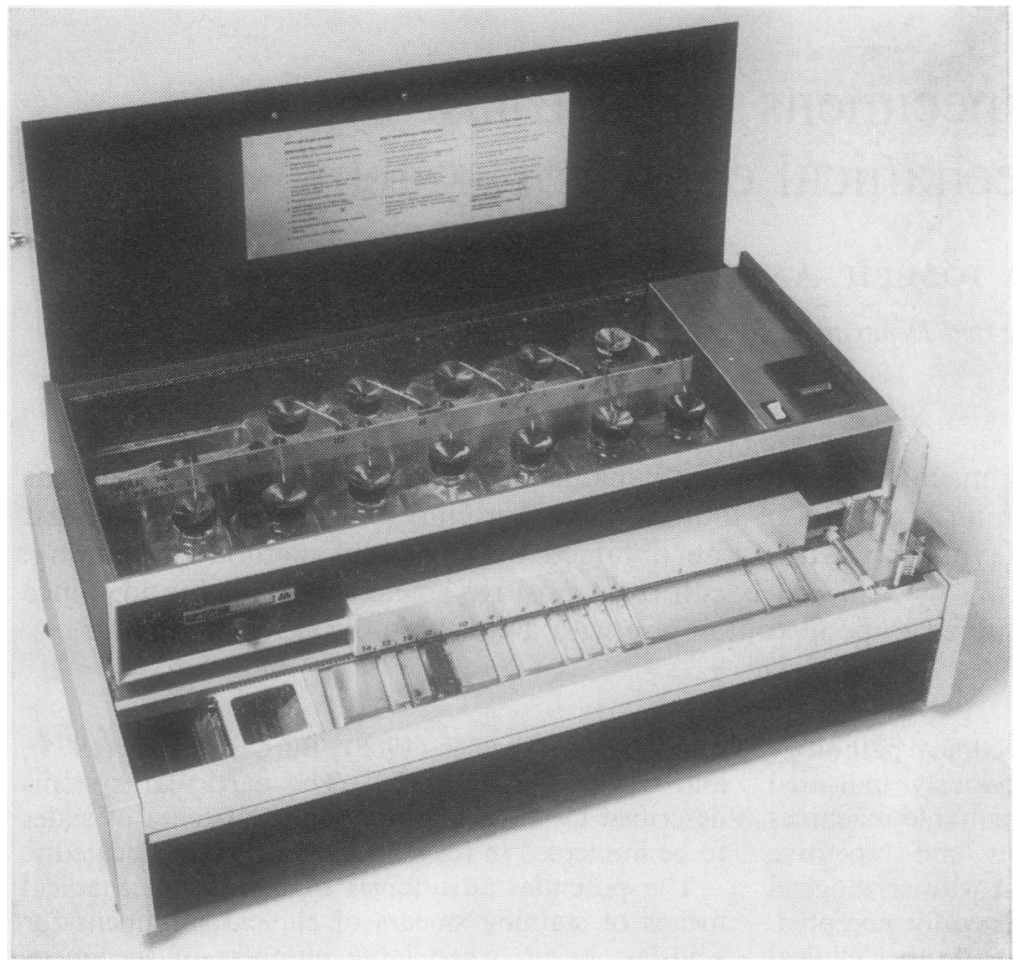

Figure The staining machine.

in a storage drawer awaiting manual removal. Stained smears are produced at a rate of 44 per hour in the first hour and thereafter at a rate of 60 per hour. It takes 16 minutes before the first slide is ready for examination.

For the purpose of staining preparations on slides with auramine the first three platen sections are connected to the reservoir containing auramine, platen sections 4-8 are connected to the reservoir containing acid-alcohol, platen sections 9 and 10 are connected to the reservoir containing distilled water, platen section 11 is connected to the reservoir containing potassium permanganate solution, and platen section 13 is connected to the reservoir containing alcohol. This results in the treatment of each preparation with the various reagents for the times given in Table 4.

Comprehensive details of the machine are given in the manufacturer's handbook.

\section{Staining procedure}

The method we have adopted for staining acid-fast bacilli by machine is based on a commonly used manual procedure for auramine staining (Cruickshank et al., 1975).
Applying reagents and measuring fluorescence emission from acid-fast bacilli as well as background fluorescence in auramine-stained smears of sputum The brightness of fluorescent acid-fast bacilli and of pus cells in smears of sputum stained manually with auramine by the method described by Cruickshank et al. (1975) was measured by a technique incorporating fibreoptics that we have developed and described earlier (Taylor and Heimer, 1974; Heimer, 1977). The measurements so obtained were used as a guide for modifying the times of applying staining solutions in the mechanical method so as to make optimum use of the machine without losing brightness in the fluorescent staining of acid-fast bacilli os or increasing background fluorescence. Table $1 \mathrm{~N}$ shows the mean of 10 photometric readings, each of $N$ which was made from an acid-fast bacillus in a fresh microscopical field. Maximum fluorescence of acid-fast bacilli was obtained after a minimum of four and a half minutes of staining with auramine.

Table 2 shows the effect of potassium permanganate solution on background fluorescence derived mainly from pus cells. Again, the figures represent the mean of 10 photometric readings. Each reading was derived from a single pus cell 
Table 1 Effect of various convenient staining times for auramine on fluorescence intensity of acid-fast bacilli

\begin{tabular}{lll}
\hline Smear & Time & $\begin{array}{l}\text { Fluorescence emission from acid-fast } \\
\text { bacilli in arbitrary units (mean of } 10 \\
\text { readings) }\end{array}$ \\
\hline 1 & $20 \mathrm{~s}$ & 15 \\
2 & $1 \mathrm{~min}$ & 28 \\
3 & $4 \frac{1}{2} \mathrm{~min}$ & 36 \\
4 & $8 \mathrm{~min}$ & 36 \\
5 & $15 \mathrm{~min}$ & 34 \\
\hline
\end{tabular}

nucleus each in a separate microscopical field. After 30 seconds' staining with the potassium permanganate solution a significant reduction in background fluorescence was obtained.

Table 2 Effect of various convenient staining times for potassium permanganate on background fluorescence as indicated by fluorescence emission from pus cell nuclei

\begin{tabular}{llll}
\hline Smear & Reagent & Time & $\begin{array}{l}\text { Fluorescence emission } \\
\text { from pus cell nuclei in } \\
\text { arbitrary units } \\
\text { (mean of } 10 \text { readings) }\end{array}$ \\
\hline 1 & Potassium permanganate & - & 100 \\
2 & Potassium permanganate & $10 \mathrm{~s}$ & 10 \\
3 & Potassium permanganate & $30 \mathrm{~s}$ & 2 \\
4 & Potassium permanganate & $1 \mathrm{~min}$ & 2 \\
5 & Potassium permanganate & $2 \mathrm{~min}$ & 2 \\
\hline
\end{tabular}

Prolonging the period of staining with potassium permanganate did not lead to any further reduction in background fluorescence but, as indicated in Table 3 , it did reduce fluorescence emission from acid-fast bacilli. Staining with potassium permanganate solution for 30 seconds had little effect on the brightness of auramine-stained tubercle bacilli.

Table 3 Effect of various convenient staining times for potassium permanganate on brightness of auraminestained tubercle bacilli

\begin{tabular}{lll}
\hline Smear & Time & $\begin{array}{l}\text { Fluorescence emission from acid-fast } \\
\text { bacilli in arbitrary units } \\
\text { (mean of } 10 \text { readings) }\end{array}$ \\
\hline 1 & - & 35 \\
2 & $10 \mathrm{~s}$ & 30 \\
3 & $30 \mathrm{~s}$ & 31 \\
4 & $1 \mathrm{~min}$ & 24 \\
\hline
\end{tabular}

As a result of these preliminary investigations we decided to use for the routine mechanical staining of sputum smears a timing sequence, as indicated in Table 4.

Testing for contamination of negative smears with acid-fast bacilli from positive smears

Ten known positive and 10 known negative smears were arranged alternately in the loading magazine
Table 4 Timing sequence for mechanical staining of sputum smears with auramine

\begin{tabular}{llll}
\hline Platen section & Reagent & \multicolumn{2}{l}{ Time } \\
\cline { 3 - 4 } & & Minutes & Seconds \\
\hline 1,2 , and 3 & Auramine & 4 & 30 \\
$4,5,6,7$, and 8 & Acid-alcohol & 3 & \\
9 and 10 & Distilled water & 1 & 30 \\
11 & Potassium permanganate & & 30 \\
12 & Nil & & 30 \\
13 & Alcohol & 1 & 30 \\
14 & Nil & \\
\hline
\end{tabular}

and stained mechanically, as indicated in Table 4. Identifying data on each slide were covered before the slides were examined in random order by two experienced observers. Acid-fast bacilli were not observed in any smear made from a known negative specimen. Contamination of negative smears with bacilli from positive smears would not therefore appear to be a serious problem, especially as reagents are dispensed freshly to each smear.

Comparing mechanical and manual methods for efficiency of staining clinical specimens for acid-fast bacilli

Two smears were made from each of 95 unselected routine specimens. One smear from each specimen was stained manually and examined microscopically, and the result was recorded. The 95 duplicate smears were stained by the machine. Slides were randomised and, after identifying data had been covered, each slide was examined microscopically. The results are shown in Table 5.

Table 5 Comparison of results after auramine staining of duplicate slides manually and by machine

\begin{tabular}{lccc}
\hline & Machine + & Machine & Total \\
\hline Manual + & 14 & 0 & 14 \\
Manual - & 3 & 173 & 176 \\
Total & 17 & 173 & 190 \\
\hline
\end{tabular}

Smears from the 14 specimens that were positive by the manual method were positive also by the machine method. In addition, smears from three specimens that were negative by the manual method were positive by the machine method. In these three specimens acid-fast bacilli were found in very small numbers.

\section{Discussion}

Suitably modified, in our hands, the Cyto-Tek staining machine seems to meet the necessary requirements for staining smears of clinical specimens for acid-fast bacilli. We have not been able to 
demonstrate any transfer of acid-fast bacilli from one slide to another by the machine.

Since the study described in this paper was undertaken the machine has been in routine use for eight months, during which time approximately 4500 slides have been satisfactorily processed with a considerable saving in labour.

\section{References}

Burdash, N. M., West, M. E., and Bannister, E. R. (1975). Automatic Gram-staining with the Microstainer II. Health Laboratory Science, 13, No. 1, 20-22.

Clancey, J. K., Allen, B. W., Rogers, D. T., Smith, L. S., Aber, V., and Mitchison, D. A. (1976). Comparison of machine and manual staining of direct smears for acid-fast bacilli by fluorescence microscopy. Journal of Clinical Pathology, 29, 931-933.
Cremer, A. W. F. (1968). Automatic slide staining in clinical bacteriology. Journal of Medical Laboratory Technology, 25, 387-390.

Cruickshank, R., Duguid, J. P., Marmion, B. P., and Swain, R. H. A. (1975). In Medical Microbiology, 12th edition, vol. 2, p. 40. Churchill Livingston, Edinburgh, London and New York.

Heimer, G. V. (1977). Simple inexpensive equipment for microfluorometry. In Rapid Methods and Automation in Microbiology, edited by H. H. Johnston, and S. W. B. Newsom, pp. 103-104. Learned Information (Europe), Oxford, England.

Taylor, C. E. D. (1969). Serological techniques. Journal of Clinical Pathology, 22, supplement, 3, 14-18.

Taylor, C. E. D., and Heimer, G. V. (1974). Measuring immunofluorescence emission in terms of standard international physical units. Journal of Biological Standardization, 2, 11-20. 